\title{
The Matching Uniqueness of A Graphs
}

\author{
Shichang Shen \\ School of Mathematics and Statistics, Qinghai Nationalities University, Xining, China \\ Email: 13909785766@163.com
}

Received 20 March 2015; accepted 30 June 2015; published 3 July 2015

Copyright (C) 2015 by author and Scientific Research Publishing Inc.

This work is licensed under the Creative Commons Attribution International License (CC BY).

http://creativecommons.org/licenses/by/4.0/

c) (i) Open Access

\section{Abstract}

In the paper, We discussed the matching uniqueness of graphs with degree sequence $\left(1^{3}, 2^{s-4}, 3\right)$. The necessary and sufficient conditions for $T(1,5, n) \cup\left(\bigcup_{i=0}^{s} C_{p_{i}}\right)$ and its complement are matching unique are given.

\section{Keywords}

\section{Graph, Matching Polynomial, Matching Uniqueness}

\section{Introduction}

All graphs considered in the paper are simple and undirected. The terminology not defined here can be found in [1]. Let $G$ be a graph with $n$ vertices. An $r$-matching in a graph $G$ is a set of $r$ edges, no two of which have a vertex in common. The number of $r$-matching in $G$ will be denoted by $p(G, r)$. We set $p(G, 0)=1$ and define the matching polynomial of $G$ by

$$
\mu(G, x)=\sum_{r \geq 0}(-1)^{r} p(G, r) x^{n-2 r}
$$

For any graph $G$, the roots of $\mu(G, x)$ are all real numbers. Assume that $\gamma_{1}(G) \geq \gamma_{2}(G) \geq \cdots \geq \gamma_{n}(G)$, the largest root $\gamma_{1}(G)$ is referred to as the largest mathing root of $G$.

Throughout the paper, we denote by $P_{n}$ and $C_{n}$ the path and the cycle on $n$ vertices, respectively. $T(a, b, c)(a \leq b \leq c)$ denotes the tree with a vertex $v$ of degree 3 such that $T(a, b, c)-v=P_{a} \cup P_{b} \cup P_{c}$, and $T(a, b, c, 1,1)(a \leq b \leq c)$ denotes the tree obtained by appending a pendant vertex of the path $P_{c}$ in $T(a, b, c)$ to a vertex with degree 2 of $P_{3} . Q\left(s_{1}, s_{2}\right)$ is obtained by appending a cycle $C_{s_{1}+1}$ to a pendant vertex of a path $P_{s_{2}}$. Two graphs are matching equivalency if they share the same matching polynomial. A graph $G$ is said to be matching unique if for any graph $H, \mu(G, x)=\mu(H, x)$ implies that $H$ is isomorphic to $G$. The study in 
this ares has made great progress. For details, the reader is referred to the surveys [2]-[6]. In the paper, we prove $T(1,5, n) \cup\left(\bigcup_{i=0}^{s} C_{p_{i}}\right)(n \geq 5)$ and its complement are matching unique if and only if $n \neq 5,8,15$ or $n=6$, $p_{i} \neq 6$.

\section{Basic Results}

Lemma 1 [1] The matching polynomial $\mu(G, x)$ satisfies the following identities:

1) $\mu(G \cup H, x)=\mu(G, x) \mu(H, x)$.

2) $\mu(G, x)=\mu(G \backslash e, x)-\mu(G \backslash u, v, x)$ if $e=\{u, v\}$ is an edge of $G$.

Lemma 2 [1] Let $G$ be a connected graph, and let $H$ be a proper subgraph $G$.

Then $\gamma_{1}(G)>\gamma_{1}(H)$.

Lemma 3 [2] Let $G=T(a, b, c) \cup\left(\bigcup_{i=0}^{s} C_{p_{i}}\right)$, if $H \sim G$, then $H$ are precisely the graphs of the following types:

$$
T\left(s_{1}, s_{2}, s_{3}\right) \cup\left(\bigcup_{i=0}^{m} C_{q_{i}}\right), Q\left(s_{1}, s_{2}\right) \cup P_{l} \cup\left(\bigcup_{i=0}^{m} C_{q_{i}}\right), K_{1} \cup\left(\bigcup_{i=0}^{m} C_{q_{i}}\right)
$$

Lemma 4 1) [1] $\gamma_{1}\left(P_{n}\right)=2 \cos \left(\frac{\pi}{n+1}\right), \gamma_{1}\left(C_{n}\right)=2 \cos \left(\frac{\pi}{2 n}\right)$.

2) [2] $\gamma_{1}(T(m, m, n))=\gamma_{1}(Q(m, n))=\gamma_{1}(Q(n+1, m-1))$.

3) [2] $\gamma_{1}(Q(2, m-1)) \leq \gamma_{1}(T(1, m, n))(2<m \leq n)<\gamma_{1}(Q(2, m+1))$.

4) [3] $\gamma_{1}(T(m, m, n))>\gamma_{1}(Q(m-1, n))(m \geq 3), \gamma_{1}(Q(m+1, m))=\gamma_{1}(Q(m, 2 m+2))$, $\gamma_{1}(Q(m, m-1))>\gamma_{1}(Q(m-1, m))$.

5) [4] $\gamma_{1}(T(1,3, n))<\gamma_{1}(T(1,4,6)), \gamma_{1}(T(1,4, n))<\gamma_{1}(T(1,5,7))$.

6) [5] $2<\gamma_{1}(T(1, m, n))(2<m<n)<(2+\sqrt{5})^{\frac{1}{2}}<\gamma_{1}\left(T\left(s_{1}, s_{2}, s_{3}\right)\right)\left(2 \leq s_{1}<s_{2}<s_{3}\right)$.

Lemma 5 [5] Let $G$ be a tree and let $G_{u, v}$ be obtained from $G$ by subdividing the edge $u v$ of $G$, then

1) $\gamma_{1}\left(G_{u, v}\right)>\gamma_{1}(G)$, if $u v$ not lies on an internal path of $G$.

2) $\gamma_{1}\left(G_{u, v}\right)<\gamma_{1}(G)$, if $u v$ lies on an internal path of $G$, and if $G$ is not isomorphic to $T(1,1, n, 1,1)$.

Lemma 6 [6] $\bigcup_{i=0}^{s} C_{p_{i}}$ are matching unique.

Lemma $7 \gamma_{1}(T(1,5, n))<\gamma_{1}(T(1,6,8))$.

Proof. Direct computation (using Matlab 8.0), we immediately have the following:

$$
\begin{gathered}
\mu(T(1,5,9,1,1), x)=x^{19}-18 x^{17}+134 x^{15}-533 x^{13}+122 x^{11}-1617 x^{9}+1176 x^{7}-413 x^{5}+50 x^{3}, \\
\mu(T(1,6,8), x)=x^{16}-15 x^{14}+90 x^{12}-276 x^{10}+458 x^{8}-400 x^{6}+164 x^{4}-24 x^{2}+1 . \\
\gamma_{1}(T(1,5,9,1,1))=2.0518, \gamma_{1}(T(1,6,8))=2.0522 .
\end{gathered}
$$

By Lemma 2, 5, we get $\gamma_{1}(T(1,5,5))<\gamma_{1}(T(1,5,6))<\gamma_{1}(T(1,5,7))<\cdots<\gamma_{1}(T(1,5, n))$

$$
<\gamma_{1}(T(1,5, n-2,1,1))<\gamma_{1}(T(1,5, n-3,1,1))<\cdots<\gamma_{1}(T(1,5,9,1,1))<\gamma_{1}(T(1,6,8)) .
$$

\section{Main Results}

Theorem 1 Let $G=T(1,5, n) \cup\left(\bigcup_{i=0}^{s} C_{p_{i}}\right)(n \geq 5)$, then $G$ are matching unique if and only if $n \neq 5,8,15$ or 
$n=6, p_{i} \neq 6$.

Proof. The necessary condition follows immediately from Lemma 1 . We have

$$
\begin{gathered}
\mu\left(T(1,5,5) \cup\left(\bigcup_{i=0}^{s} C_{p_{i}}\right), x\right)=\mu\left(Q(5,1) \cup P_{5} \cup\left(\bigcup_{i=0}^{s} C_{p_{i}}\right), x\right) \\
\mu\left(T(1,5,8) \cup\left(\bigcup_{i=0}^{s} C_{p_{i}}\right), x\right)=\mu\left(Q(2,5) \cup P_{7} \cup\left(\bigcup_{i=0}^{s} C_{p_{i}}\right), x\right) \\
\mu\left(T(1,5,15) \cup\left(\bigcup_{i=0}^{s} C_{p_{i}}\right), x\right)=\mu\left(T(1,6,7) \cup C_{7} \cup\left(\bigcup_{i=0}^{s} C_{p_{i}}\right), x\right) \\
\mu\left(T(1,5,6) \cup C_{6} \cup\left(\bigcup_{i=0}^{s} C_{p_{i}}\right), x\right)=\mu\left(T(1,4,13) \cup\left(\bigcup_{i=0}^{s} C_{p_{i}}\right), x\right)
\end{gathered}
$$

Now suppose that $n \neq 5,8,15$ or $n=6, p_{i} \neq 6, H$ is a graph being matching equivalency with $G$. We proceed to prove that $H$ must be isomorphic to $G$. By Lemma 3

$$
H \in\left\{T\left(s_{1}, s_{2}, s_{3}\right) \cup\left(\bigcup_{i=0}^{m} C_{q_{i}}\right), Q\left(s_{1}, s_{2}\right) \cup P_{l} \cup\left(\bigcup_{i=0}^{m} C_{q_{i}}\right), K_{1} \cup\left(\bigcup_{i=0}^{m} C_{q_{i}}\right)\right\}
$$

Case 1. If $H=Q\left(s_{1}, s_{2}\right) \cup P_{l} \cup\left(\bigcup_{i=0}^{m} C_{q_{i}}\right)$. By $n>5$, we know that $\gamma_{1}(H)>2$. Hence, the component of $\gamma_{1}(H)>2$ in $H$ may be only $Q\left(s_{1}, s_{2}\right)$. By Lemma $4, \gamma_{1}(Q(2,4)) \leq \gamma_{1}(T(1,5, n))<\gamma_{1}(Q(2,6))$ and $\gamma_{1}(T(1,5,8))=\gamma_{1}(Q(2,5))$. Let $s_{1}=2$, then $\gamma_{1}(T(1,5, n)) \neq \gamma_{1}\left(Q\left(2, s_{2}\right)\right)$, a contradiction. Let $s_{1}=3$. If $s_{2}=1$, then $\gamma_{1}(Q(3,1))=\gamma_{1}(Q(2,2))$, a contradiction. If $s_{2}=2$, then $\gamma_{1}(Q(3,2))=\gamma_{1}(Q(2,6))$, a contradiction. If $s_{2} \geq 3$, then $\gamma_{1}\left(Q\left(3, s_{2}\right)\right) \geq \gamma_{1}(Q(3,3))>\gamma_{1}(Q(2, n))$, a contradiction. Let $s_{1}=4$. If $s_{2}=1$, then $\gamma_{1}(Q(4,1))=\gamma_{1}(Q(2,3))$, a contradiction. If $s_{2} \geq 2$, then $\gamma_{1}\left(Q\left(4, s_{2}\right)\right) \geq \gamma_{1}(Q(4,2))=\gamma_{1}(Q(3,3))>\gamma_{1}(Q(2, n))$, a contradiction. Let $s_{1}=5$. If $s_{2}=1$, then $\gamma_{1}(Q(5,1))=\gamma_{1}(Q(2,4))$, a contradiction. If $s_{2} \geq 2$, then $\gamma_{1}\left(Q\left(5, s_{2}\right)\right) \geq \gamma_{1}(Q(5,2))=\gamma_{1}(Q(3,4))>\gamma_{1}(Q(3,3))>\gamma_{1}(Q(2, n))$, a contradiction. Let $s_{1} \geq 6$, then $\gamma_{1}\left(Q\left(s_{1}, s_{2}\right)\right) \geq \gamma_{1}(Q(6,1))>\gamma_{1}(Q(2,5))$, a contradiction.

Case 2 If $H=T\left(s_{1}, s_{2}, s_{3}\right) \cup\left(\bigcup_{i=0}^{m} C_{q_{i}}\right)$. By $\gamma_{1}(H)>2$, hence the component of $\gamma_{1}(H)>2$ in $H$ may be only $T\left(s_{1}, s_{2}, s_{3}\right)$. Let $s_{1}=1$. If $s_{2}=1$, then $\gamma_{1}\left(T\left(1,1, s_{3}\right)\right)<2$, a contradiction. If $s_{2}=2,3$, then $\gamma_{1}\left(T\left(1,2, s_{3}\right)\right)<\gamma_{1}(Q(2,4))$, a contradiction. If $s_{2}=4$, then $\gamma_{1}\left(T\left(1,4, s_{3}\right)\right)=\gamma_{1}(T(1,5, n))$, by Lemma 4 , we get $n=6$, thus $s_{3}=13$. That is,

$$
\begin{aligned}
& \mu\left(T(1,5,6) \cup C_{6} \cup\left(\bigcup_{i=0}^{m} C_{q_{i}}\right), x\right)=\mu\left(T(1,4,13) \cup\left(\bigcup_{i=0}^{m} C_{q_{i}}\right), x\right)=\mu\left(T(1,5,6) \cup\left(\bigcup_{i=0}^{s} C_{p_{i}}\right), x\right), \text { then } \\
& \mu\left(C_{6} \cup\left(\bigcup_{i=0}^{m} C_{q_{i}}\right), x\right)=\mu\left(\bigcup_{i=0}^{s} C_{p_{i}}, x\right), \text { by Lemma } 6, p_{i} \text { has at least one equal to 6, a contradiction. If } s_{2}=5,
\end{aligned}
$$
by Lemma 4, 6, we have $s_{3}=n, s=m, p_{i}=q_{i}$, thus $H$ be isomorphic to $G$. Let $s_{1}=2$. If $s_{2}=2$, $\gamma_{1}\left(T\left(2,2, s_{3}\right)\right)=\gamma_{1}\left(Q\left(2, s_{3}\right)\right) \neq \gamma_{1}(T(1,5, n))$, a contradiction. If $s_{2} \geq 3, s_{3} \geq 3$, a contradiction. Let $s_{1} \geq 3$, by Lemma 4, $\gamma_{1}(T(1,5, n))<\gamma_{1}\left(T\left(s_{1}, s_{2}, s_{3}\right)\right)$, a contradiction.

Case 3 If $H=K_{1} \cup\left(\bigcup_{i=0}^{m} C_{q_{i}}\right)$, by $\gamma_{1}(G)>2$, a contradiction. Combing cases $1-3, H$ is isomorphic to $G$. 
The proof is complete. For a graph, its matching polynomial determine the matching polynomial of its Complement [6], so the complement of $G=T(1,5, n) \cup\left(\bigcup_{i=0}^{s} C_{p_{i}}\right)(n \geq 5)$ are matching unique if and only if $n \neq 5,8,15$ or $n=6, p_{i} \neq 6$.

\section{References}

[1] Godsil, C.D. (1993) Algebraic Combinatorics. Chapman and Hall, New York, London.

[2] Shen, S.C. (2001) A Necessary and Sufficient Conditions for Matching Uniqueness of a Class of T-Shape tree. Journal of Mathematical Study, 34, 411-416.

[3] Ma, H.C. (2003) The Matching Equivalent Classes of Graphs with the Maximum Root Less than 2. Journal of Systems Science and Mathematical Sciences, 3, 337-342.

[4] Cvetkovic, D.M., Doob, M. and Sachs, H. (1980) Spectra of Graphs. Academic Press, New York.

[5] Ghareghani, N., Omidi, G.R. and Tayfeh-Rezaie, B. (2007) Spectral Characterization of Graphs with Index at Most $\sqrt{2+\sqrt{5}}$. Linear Algebra and Its Applications, 420, 483-489. http://dx.doi.org/10.1016/j.laa.2006.08.009

[6] Beezet, R.A. and Farrell, E.J. (1995) The Matching Polynomials of a Regular Graphs. Discrete Mathematics, 137, 718. 